\title{
Sampling Plan for Assaying Plates Containing Depleted or Normal Uranium
}

\author{
Ivan R. Thomas
}

November 2011

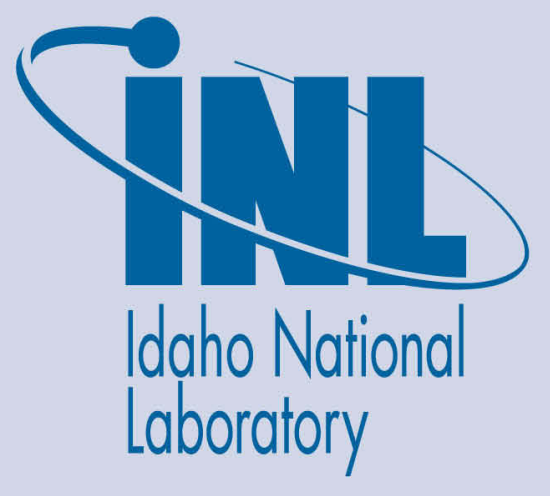

The INL is a U.S. Department of Energy National Laboratory operated by Battelle Energy Alliance 
INL/EXT-11-23828

\section{Sampling Plan for Assaying Plates Containing Depleted or Normal Uranium}

Ivan R. Thomas

November 2011

Idaho National Laboratory Idaho Falls, Idaho 83415

http://www.inl.gov

Prepared for the U.S. Department of Energy 


\section{Sampling Plan for Assaying Plates Containing Depleted or Normal Uranium}

Ivan R. Thomas, Idaho National Laboratory

\section{Introduction}

This paper describes the rationale behind the proposed method for selecting a "representative" sample of uranium metal plates, portions of which will be destructively assayed at the Y-12 Security Complex. The total inventory of plates is segregated into two populations, one for Material Type 10 (depleted uranium (DU)) and one for Material Type 81 (normal [or natural] uranium (NU)). The plates within each population are further stratified by common dimensions. A spreadsheet gives the collective mass of uranium element (and isotope for DU) and the piece count of all plates within each stratum. These data are summarized in Table 1.

Table 1 DU and NU Summary Data

\begin{tabular}{|r|r|r|r|r|r|}
\hline Uranium Type & Number of Strata & Metric Tons (Element) & \multicolumn{3}{|c|}{ Piece Count } \\
\hline Depleted & 22 & 148.2 & $99 \%$ & 352036 & $95 \%$ \\
\hline Normal & 6 & 1.1 & $1 \%$ & 17995 & $5 \%$ \\
\hline
\end{tabular}

All plates are $100 \%$ uranium metal, and all but approximately $60 \%$ of the NU plates have Kel-F® coating. The book inventory gives an overall U-235 isotopic percentage of $0.22 \%$ for the DU plates, ranging from $0.19 \%$ to $0.22 \%$. The U-235 ratio of the NU plates is assumed to be $0.71 \%$. As shown in Table 1, the vast majority of the plates are comprised of depleted uranium, so most of the plates will be sampled from the DU population.

\section{Sample Size Specification}

With the exception of plate dimensions (and the absence of Kel-F® coating on about $60 \%$ of the NU plates), all plates within each population can be considered to be homogeneous. Thus, provided that a uniform method is used for extracting portions of the plates for destructive assay, the resulting analyses should indicate minimal variability, regardless of the dimensions of plates that are sampled. Minimal variability in the uranium composition will simplify the sampling process, and each population could conceivably be sampled via a simple random sample. A simple random sample is such that all possible samples of the same size (drawn from a finite population) would have an equal probability of selection. Unfortunately, the drawing of a simple random sample from either population would be impractical, due to the large number of plates on inventory. However, the concept of a single sample will lead to determining the necessary sample size.

A formal determination of the number of items to be randomly selected is based upon three components, two of which are subjective criteria that must be specified before the sample is drawn, and the third is an estimate of the population variance. The first criterion is a "tolerable" difference between the estimated (measured) average uranium per plate and the actual but unknown average uranium for the entire population. The second criterion is a "desired" level of confidence (e.g., 95\%) that the difference 
between the estimated and actual averages is no greater than the specified tolerable difference. These criteria have not been specified.

The third component, an estimate of the statistical variance, is unavailable. As with the average content of uranium in all plates, the variance is generally unknown and must be estimated through the destructive assay of the plates that are sampled. Without the specified criteria and lacking a defensible estimate of variance (that is based upon expert judgment or other information aside from destructive assay), the classical method for determining the sample size cannot be employed. Consequently, the sample size for each population will be based upon a number that seems intuitively representative of the population and which is operationally feasible.

If little plate-to-plate variability is present, then a small sample should yield an adequate estimate of the population average, whereas greater plate-to-plate variability would require a larger sample size. A sample of size 30 is traditionally regarded as being "sufficiently large" for estimating the parameters of a large population, especially if there is little variability in the individual items, as should be true for the uranium composition of all plates. Consequently, the combined sample size will be arbitrarily set at 34 plates, of which 30 will come from the larger DU population, and 4 will from NU population, which only accounts for $1 \%$ of the combined mass of uranium and only $5 \%$ of the total number of plates.

\section{$\underline{\text { Population Stratification }}$}

As noted, the drawing of a simple random sample from an entire population would be operationally infeasible. A more tractable approach would involve sub-sampling the individual strata, with the largest strata being assigned the largest number of subsamples. The combined sample for the entire population would then be the sum of the separate subsamples. Thus, for both populations, the sampling plan must first identify the strata to be sampled and the number of plates (if any) to be sampled per strata. Lastly, the plan must then describe how the plates will be selected from each stratum.

The size of each subsample will be based upon the quantity of material (both piece count and mass) within the stratum. The piece count of the DU strata dramatically ranges from 2 to 170508, and the mass ranges from roughly 0 to 66.0 metric tons. The piece count and masses of the NU population are not as variable, having respective ranges of 1178 to 5500 plates and 0.03 to 0.6 metric tons. The observed variability is compounded by the differing plate dimensions, which causes the cumulative mass per stratum to be un-proportional to the corresponding piece count. Inasmuch as neither piece count nor cumulative mass can be considered a more significant metric for uranium quantity, both will be used for prorating the subsamples per strata. The final proration will be the average of the subsamples obtained using both methods.

\section{$\underline{\text { Stratum Sample Selection }}$}

The first proration is based upon piece count, and the strata are randomly selected using a classical sampling technique called Probability Proportional to Size (PPS). With this method, the probability of a plate being drawn in a subsample is proportional to the number of plates in the stratum. The individual strata are delineated by upper and lower boundaries of the cumulative piece count. The lower boundary for the first stratum is one, with the upper boundary being the number of plates in the first stratum. The lower boundary of the second stratum is the number of plates in the first stratum plus one, and the upper 
boundary is the sum of the number of plates in the first two strata. Likewise, the lower boundary of the third stratum is the upper boundary of the second stratum plus one, and the upper boundary is the sum of the plates in the first three strata. This delineation continues on to the last stratum, having an upper boundary equal to the grand total of all plates in the population. For the DU population, a series of 30 random integers was generated, ranging from one to the total piece count (352036). For every generated random integer enclosed within the defined boundaries of a given stratum, a plate was included in that stratum's subsample. Using this method of proration, the size of each subsample is an integer. Four additional random numbers were generated for the NU population, ranging from one to the total piece count (17995), with the size of the subsamples being determined in the same fashion as for the DU population.

The second method of proration did not include the random selection of strata but was based strictly upon mass. The size of the stratum subsamples was obtained by multiplying the total sample size ( 34 for the DU population and 4 for the NU population) by the ratio of the stratum mass to the collective mass of the entire population. Unlike the proration using piece count, the size of each subsample is not an integer. Thus, the number 34, rather than 30, was used as an "inflation" multiplier for the DU population; otherwise, the rounding process would have reduced the total sample size to 28 , rather than the predetermined value of 30 .

The sizes of the stratum subsamples shown in Table 2 and Table 3 were obtained by averaging the number of subsamples obtained by the two proration methods, with the result being rounded to an integer.

Table 2 DU Population

\begin{tabular}{|c|c|c|}
\hline Stratum & Plate Dimensions & Subsample Size \\
\hline 1 & $0.0625 " \times 2 " \times 3 "$ & 1 \\
\hline 2 & $0.0625 " \times 2 " \times 2 "$ & 0 \\
\hline 3 & $0.0625 " \times 1 " \times 2 "$ & 0 \\
\hline 4 & $0.0625 " \times 1 " \times 1 "$ & 0 \\
\hline 5 & $0.0625 " \times 0.5 " x 2 "$ & 0 \\
\hline 6 & $0.125 " x$ 1"x 1" & 0 \\
\hline 7 & $0.125 " \times 0.5 " x 1 "$ & 0 \\
\hline 8 & $0.125 " \times 0.5 " \times 2 "$ & 0 \\
\hline 9 & $0.125 " \times 2 " x$ 1" & 2 \\
\hline 10 & $0.125 " \times 2 " x$ 2" & 10 \\
\hline 11 & $0.125 " \times 2 " x$ 3" & 5 \\
\hline 12 & $0.125 " \times 2 " x 4 "$ & 0 \\
\hline 13 & $0.125 " \times 2 " \times 5 "$ & 0 \\
\hline 14 & $0.125 " \times 2 " x$ 6" & 0 \\
\hline 15 & $0.125 " \times 2 " \times 7 "$ & 0 \\
\hline 16 & $0.125 " \times 2 " x 8 "$ & 0 \\
\hline 17 & $1 " x 1 " x 5 "$ & 1 \\
\hline 18 & $1 " \times 1 " \times 1 "$ & 0 \\
\hline 19 & $1 " \times 1 " \times 2 "$ & 0 \\
\hline 20 & $1 " \times 1 " \times 3 "$ & 0 \\
\hline 21 & $2 " x 2 " x 2 "$ & 2 \\
\hline 22 & $2 " x 2 " x$ 5" & $\underline{9}$ \\
\hline & Total Sample Size & 30 \\
\hline
\end{tabular}


Table 3 NU Population

\begin{tabular}{|c|c|c|}
\hline Stratum & Plate Dimensions & Subsample Size \\
\hline 1 & $0.125 " \times 0.5 " \times 2 "$ & 0 \\
\hline 2 & $0.125 " x$ 1"x 2" & 1 \\
\hline 3 & $0.125 " \times 2 " x$ 2" & 1 \\
\hline 4 & $0.03125 " x$ 2" x 3" * & 1 \\
\hline 5 & $0.03125 " x$ 2" x 2" * & 1 \\
\hline 6 & $0.03125 ” \times 2 " \times 1 " *$ & $\underline{\underline{0}}$ \\
\hline & Total Sample Size & 4 \\
\hline
\end{tabular}

*Not Kel-F® Coated

The only concern with the above sampling approach is that some of the NU plates ( $60 \%$ by piece count and $34 \%$ by mass) do not have the Kel-f® coating, which could slightly alter the reported uranium concentrations for the uncoated plates. Fortunately, of the four plates to be selected from the NU population, two will come from strata containing uncoated plates and two will come from strata containing coated plates. The equal division of subsamples should yield an overall representation of the plates within the comparatively small NU population.

\section{$\underline{\text { Plate Selection }}$}

Given the subsamples listed above, the specified number of plates must next be selected from the identified strata. Ideally, the plates would be selected at random, but a formal means of random selection is not operationally feasible. First, the plates are stored in "ammo" boxes that are stacked such that some of the boxes are not readably accessible. Second, the plates do not have serial numbers, so once an ammo box is opened, the contained plates are not individually identifiable. Consequently, the specific plates (most likely drawn from the top layer of each box) will be selected at the discretion of the operator. This approach is sanctioned, even though "arbitrary" selection is not technically the same as "random" selection. As noted, however, all plates of a particular population (DU or NU) should have the same uranium composition, regardless of the plate dimensions defining the separate strata. The uniformity of construction should imply little variability in the analytical results, so the arbitrary selection of the specified number of plates from the identified strata should yield a representative sample of all plates within each population. 